\author{
F. Hosseini Shekarabi · M. Khodabin · K. Maleknejad
}

\title{
Application of operational matrices to numerical solution of stochastic SIR model
}

Received: 7 May 2015 / Accepted: 29 January 2016 / Published online: 19 February 2016

(C) The Author(s) 2016. This article is published with open access at Springerlink.com

\begin{abstract}
The goal of this paper is to give useful method for solving a problem in biologic system that is formulated by stochastic Volterra integral equations. Here, we consider triangular functions, block pulse functions and their operational matrices of integration. Illustrative example is included to demonstrate the validity and applicability of the operational matrices.
\end{abstract}

Mathematics Subject Classification 65C30 $60 \mathrm{H} 35 \cdot 65 \mathrm{C} 20 \cdot 60 \mathrm{H} 20 \cdot 37 \mathrm{~N} 25$

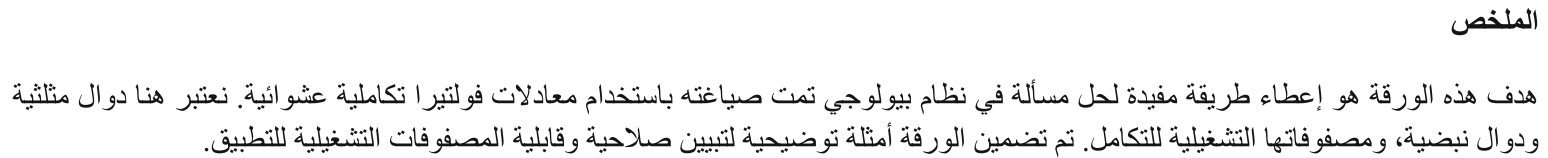

\section{Introduction}

Studies in economics, sociology, various biological and medical models lead to the stochastic Volterra integral equations (SVIEs). These systems are dependent on a noise source, a Gaussian white noise, so that modeling such phenomena naturally requires the use of various stochastic Volterra integral equations. Here, we consider stochastic SIR model.

In many fields of science, engineering and various physical phenomena there is a large number of problems which are intrinsically nonlinear and complex in nature, involving stochastic excitations of a Gaussian white noise type. In these various physical phenomena, some quantity is constantly undergoing small, random fluctuations. Having in mind that a Gaussian white noise mathematically described as a formal derivative of a Brownian motion process, all such problems are mathematically modeled by stochastic differential equations, or in more complicated cases, by stochastic Volterra integro-differential (SVID) equations of the Ito type.

In modeling the spread process of infectious diseases, many classical epidemic models have been proposed and studied, such as SIR, SEIR and SIRS models. The SIR infections disease model is an important biologic model and has been studied by many authors [6-8,11,16-19]. Epidemiology is the study of the spread of diseases with the objective to trace factors that are responsible for or contribute to their occurrence. Significant progress has been made in the theory and application of epidemiology modeling by mathematical research.

Triangular functions (TFs) were introduced by Deb et al. [5]. TF approximation has been successfully used to analysis of dynamic systems [5], variational problems [1], integral equations [2], integro-differential equations [3], and Volterra-Fredholm integral equations [4].

F. Hosseini Shekarabi $(\varangle) \cdot$ M. Khodabin $\cdot$ K. Maleknejad

Department of Mathematics, Karaj Branch, Islamic Azad University, Karaj, Iran

E-mail: f_hosseini@srttu.edu; m-khodabin@kiau.ac.ir; maleknejad@iust.ac.ir 
The numerical study and simulation of stochastic Volterra integral equations have been an active field of research for the last years $[9,12]$. Most SVIEs do not have analytic solutions and hence it is of great importance to provide numerical schemes. The paper [12] solved stochastic Volterra integral equations by stochastic operational matrix based on block pulse functions (BPFs). Also, [9] considered stochastic operational matrix of triangular functions.

In the next section, stochastic concepts of Brownian motion and Ito integral are presented. In Sect. 3, we review block pulse functions, triangular functions and their operational matrices of integration both in the deterministic and stochastic case. In Sect. 4, mathematical model of SIR is presented, then these operational matrices are applied to solve stochastic SIR model and numerical results are shown. Finally, Sect. 5 provides a brief conclusion of this work.

\section{Stochastic concepts of Ito integral}

Definition 2.1 (Brownian motion process) A real-valued stochastic process $\{B(t), t \geq 0\}$ is called Brownian motion, if it satisfies the following properties:

(i) (Independence of increments) $B(t)-B(s), t>s$, is independent of the past, that is, of $B(u), 0 \leq u \leq s$, or of $\mathcal{F}_{s}$, the $\sigma$-field generated by $B(u), u \leq s$.

(ii) (Normal increments) $B(t)-B(s)$ has Normal distribution with mean 0 and variance $t-s$.

(iii) (Continuity of paths) $B(t), t \geq 0$ are continuous functions of $t$.

Definition 2.2 Let $\{N(t)\}_{t \geq 0}$ be an increasing family of $\sigma$-algebras of sub-sets of $\Omega$. A process $g(t, \omega)$ from $[0, \infty) \times \Omega$ to $R^{n}$ is called $N(t)$-adapted if for each $t \geq 0$ the function $\omega \longrightarrow g(t, \omega)$ is $N(t)$-measurable [15].

Definition 2.3 Let $v=v(S, T)$ be the class of functions $f(t, \omega):[0, \infty) \times \Omega \longrightarrow R$ such that,

(i) $(t, \omega) \longrightarrow f(t, \omega)$ is $B \times \mathcal{F}$-measurable, where $B$ denotes the Borel $\sigma$-algebra on $[0, \infty)$ and $\mathcal{F}$ is the $\sigma$-algebra on $\Omega$.

(ii) $f(t, \omega)$ is $\mathcal{F}_{t}$-adapted, where $\mathcal{F}_{t}$ is the $\sigma$-algebra generated by the random variables $B(s) ; s \leq t$.

(iii) $E\left[\int_{S}^{T} f^{2}(t, \omega) \mathrm{d} t\right]<\infty$.

Proof See [15].

Definition 2.4 (The Itô integral) [15] Let $f \in v(S, T)$. Then the Itô integral of $f$ (from $\mathrm{S}$ to $\mathrm{T}$ ) is defined by

$$
\int_{S}^{T} f(t, \omega) \mathrm{d} B(t)(\omega)=\lim _{n \rightarrow \infty} \int_{S}^{T} \phi_{n}(t, \omega) \mathrm{d} B(t)(\omega), \quad\left(\text { limit in } L^{2}(P)\right)
$$

where $\phi_{n}$ is a sequence of elementary functions such that

$$
E\left[\int_{S}^{T}\left(f(t, \omega)-\phi_{n}(t, \omega)\right)^{2} d \mathrm{t}\right] \rightarrow 0, \quad \text { as } n \rightarrow \infty .
$$

Theorem 2.5 (The Itô isometry) Let $f \in v(S, T)$. Then

$$
E\left[\left(\int_{S}^{T}(f(t, \omega) d B(t)(w))^{2}\right)\right]=E\left[\int_{S}^{T} f^{2}(t, \omega) d(t)\right] .
$$

Proof See [15].

Definition 2.6 (one-dimensional Itô processes) [15] Let $B(t)$ be one-dimensional Brownian motion on $(\Omega, \mathcal{F}, P)$. A one-dimensional Itô process (stochastic integral) is a stochastic process $X(t)$ on $(\Omega, \mathcal{F}, P)$ of the form

$$
X(t)=X(0)+\int_{0}^{t} u(s, \omega) \mathrm{d} s+\int_{0}^{t} v(s, \omega) \mathrm{d} B(s),
$$

or

$$
\mathrm{d} X(t)=u \mathrm{~d} t+v \mathrm{~d} B(t)
$$


where

$$
\begin{gathered}
P\left[\int_{0}^{t} v^{2}(s, \omega) \mathrm{d} s<\infty, \text { for all } t \geq 0\right]=1, \\
P\left[\int_{0}^{t}|u(s, \omega)| \mathrm{d} s<\infty, \text { for all } t \geq 0\right]=1 .
\end{gathered}
$$

Theorem 2.7 (The one-dimensional Itô formula) Let $X(t)$ be an Itô process given by (1) and $g(t, x) \in$ $C^{2}([0, \infty) \times R)$. Then

$$
Y(t)=g(t, X(t))
$$

is again an Itô process, and

$$
d Y(t)=\frac{\partial g}{\partial t}(t, X(t)) d t+\frac{\partial g}{\partial x}(t, X(t)) d X(t)+\frac{1}{2} \frac{\partial^{2} g}{\partial x^{2}}(t, X(t))(d X(t))^{2},
$$

where $(d X(t))^{2}=(d X(t))(d X(t))$ is computed according to the rules

$$
\mathrm{d} t \cdot \mathrm{d} t=\mathrm{d} t \cdot \mathrm{d} B(t)=\mathrm{d} B(t) \cdot \mathrm{d} t=0, \quad \mathrm{~d} B(t) \cdot \mathrm{d} B(t)=\mathrm{d} t .
$$

Proof See [15].

Some properties of the Itô integral are listed as follows. Let $f, g \in v(0, T)$ and let $0 \leq S<U<T$. Then

1. $\int_{S}^{T} f(t) \mathrm{d} B(t)=\int_{S}^{U} f(t) \mathrm{d} B(t)+\int_{U}^{T} f(t) \mathrm{d} B(t)$

2. $\int_{S}^{T}(c f(t)+g(t)) \mathrm{d} B(t)=c \int_{S}^{T} f(t) \mathrm{d} B(t)+\int_{S}^{T} g(t) \mathrm{d} B(t)$

3. $E\left(\int_{S}^{T} f(t) \mathrm{d} B(t)\right)=0$

4. $\int_{S}^{T} f(t) \mathrm{d} B(t)$ is $F_{t}$ measurable.

For more details see $[10,15]$.

\section{Operational matrix of integration for some typical functions}

The goal of this section is to recall notations and definitions of some typical functions that are used in the next sections. Their properties and operational matrices are reviewed.

\subsection{Block pulse functions (BPFs)}

BPFs have been variously studied $[13,14]$ and applied for solving different problems.

The BPFs are defined on the time interval $[0, T)$ by

$$
\psi_{i}(t)= \begin{cases}1 & (i-1) \frac{T}{m} \leq t<i \frac{T}{m} \\ 0 & \text { elsewhere }\end{cases}
$$

where $i=1, \ldots, m$ and for convenience we put $h=\frac{T}{m}$.

The BPFs on $[0, T)$ have disjointness, orthogonality and completeness property.

The set of BPFs can be written as a vector

$$
\Psi(t)=\left[\psi_{1}(t), \ldots, \psi_{m}(t)\right]^{T} \quad t \in[0, T) .
$$

From the above representation and disjointness property, it follows that

$$
\Psi(t) \Psi^{T}(t)=\left(\begin{array}{ccccc}
\psi_{1}(t) & 0 & 0 & \cdots & 0 \\
0 & \psi_{2}(t) & 0 & \cdots & 0 \\
\vdots & \vdots & \vdots & \ddots & \vdots \\
0 & 0 & 0 & \cdots & \psi_{m}(t)
\end{array}\right)_{m \times m}
$$




$$
\begin{gathered}
\Psi^{T}(t) \Psi(t)=1, \\
\Psi(t) \Psi^{T}(t) F=\tilde{F} \Psi(t),
\end{gathered}
$$

where $F$ is a m-dimensional vector and $\tilde{F}=\operatorname{diag}(F)$. The expansion of a function $f(t)$ over $[0, T)$ with respect to $\psi_{i}(t), i=1, \ldots, m$ is given by

$$
f(t) \simeq \sum_{i=1}^{m} f_{i} \psi_{i}(t)=F^{T} \Psi(t)=\Psi^{T}(t) F,
$$

where $F=\left[f_{1}, \ldots, f_{m}\right]^{T}$ and $f_{i}$ is

$$
f_{i}=\frac{1}{h} \int_{0}^{T} f(t) \psi_{i}(t) \mathrm{d} t
$$

Now, operational matrix of integration is considered

$$
\int_{0}^{t} \Psi(s) \mathrm{d} s \simeq Q \Psi(t)
$$

where $Q$ is operational matrix of integration that is given by

$$
Q=\frac{h}{2}\left(\begin{array}{ccccc}
1 & 2 & 2 & \cdots & 2 \\
0 & 1 & 2 & \cdots & 2 \\
0 & 0 & 1 & \cdots & 2 \\
\vdots & \vdots & \vdots & \ddots & \vdots \\
0 & 0 & 0 & \cdots & 1
\end{array}\right)
$$

So,

$$
\int_{0}^{t} f(s) \mathrm{d} s \simeq \int_{0}^{t} F^{T} \Psi(s) \mathrm{d} s \simeq F^{T} Q \Psi(t)
$$

The Itô integral of BPFs is defined by

$$
\int_{0}^{t} \Phi(s) \mathrm{d} B(s) \simeq Q_{S} \Phi(t)
$$

where stochastic operational matrix of integration is given by

$$
Q_{S}=\left(\begin{array}{ccclc}
B\left(\frac{h}{2}\right) & B(h) & B(h) & \cdots & B(h) \\
0 & B\left(\frac{3 h}{2}\right)-B(h) & B(2 h)-B(h) & \cdots & B(2 h)-B(h) \\
0 & 0 & B\left(\frac{5 h}{2}\right)-B(2 h) & \cdots & B(3 h)-B(2 h) \\
\vdots & \vdots & \vdots & \ddots & \vdots \\
0 & 0 & 0 & \cdots & B\left(\frac{(2 m-1) h}{2}\right)-B((m-1) h)
\end{array}\right)_{m \times m}
$$

So, the Itô integral of every function $f(t)$ can be approximated by

$$
\int_{0}^{t} f(s) \mathrm{d} B(s) \simeq \int_{0}^{t} F^{T} \Phi(s) \mathrm{d} B(s) \simeq F^{T} Q_{S} \Phi(t) .
$$


3.2 Triangular functions (TFs)

Two m-sets of triangular functions are defined [5] over the interval $[0, T)$ as

$$
\begin{gathered}
T_{i}^{1}(t)= \begin{cases}1-\frac{t-i h}{h} & i h \leq t<(i+1)) h, \\
0 & \text { elsewhere }\end{cases} \\
T_{i}^{2}(t)= \begin{cases}\frac{t-i h}{h} & i h \leq t<(i+1)) h, \\
0 & \text { elsewhere }\end{cases}
\end{gathered}
$$

where $i=0, \ldots, m-1$ and $m$ is the number of elementary functions and $h=\frac{T}{m}$. Moreover,

$$
T_{i}^{1}(t)+T_{i}^{2}(t)=\psi_{i}(t)
$$

From the definition of TFs, it is clear that TFs are disjoint, orthogonal, and complete [5].

We can write TFs in vector form

$$
\begin{aligned}
& T 1(t)=\left[T_{0}^{1}(t), \ldots, T_{m-1}^{1}(t)\right]^{T}, \\
& T 2(t)=\left[T_{0}^{2}(t), \ldots, T_{m-1}^{2}(t)\right]^{T},
\end{aligned}
$$

and

$$
T(t)=[T 1(t), T 2(t)]^{T},
$$

where $T(t)$ is called the TF vector.

Put

$$
T(t) \cdot T^{T}(t) \simeq \operatorname{diag}(T(t)),
$$

where $\operatorname{diag}(T(t))$ is a $2 m \times 2 m$ diagonal matrix.

Furthermore,

$$
T(t) T^{T}(t) F \simeq \tilde{F} T(t),
$$

where $F$ is a $2 m$-dimensional vector and $\tilde{F}=\operatorname{diag}(F)$ is $2 m \times 2 m$ diagonal matrix.

The expansion of $f(t)$ over $[0, T)$ with respect to $1 \mathrm{D}-\mathrm{TFs}$, is given by

$$
\begin{aligned}
f(t) & \simeq \sum_{i=0}^{m-1} C 1_{i} T_{i}^{1}(t)+\sum_{i=0}^{m-1} C 2_{i} T_{i}^{2}(t)=C 1^{T} \cdot T 1(t)+C 2^{T} \cdot T 2(t) \\
& =[C 1, C 2]^{T} .[T 1(t), T 2(t)]=C^{T} . T(t),
\end{aligned}
$$

where $C 1_{i}$ and $C 2_{i}$ are samples of $f$, for example, $C 1_{i}=f(i h)$ and $C 2_{i}=f((i+1) h)$ for $i=0,1, \ldots, m-1$. The vector $C$ is called the TF coefficient vector.

Consider

$$
\begin{aligned}
& \int_{0}^{t} T 1(s) \mathrm{d} s=M_{1} \cdot T 1(t)+M_{2} \cdot T 2(t), \\
& \int_{0}^{t} T 2(s) \mathrm{d} s=M_{1} \cdot T 1(t)+M_{2} \cdot T 2(t),
\end{aligned}
$$

where

$$
M 1=\frac{h}{2}\left(\begin{array}{ccccc}
0 & 1 & 1 & \cdots & 1 \\
0 & 0 & 1 & \cdots & 1 \\
0 & 0 & 0 & \cdots & 1 \\
\vdots & \vdots & \vdots & \ddots & \vdots \\
0 & 0 & 0 & \cdots & 0
\end{array}\right)_{m \times m}
$$




$$
M 2=\frac{h}{2}\left(\begin{array}{ccccc}
1 & 1 & 1 & \cdots & 1 \\
0 & 1 & 1 & \cdots & 1 \\
0 & 0 & 1 & \cdots & 1 \\
\vdots & \vdots & \vdots & \ddots & \vdots \\
0 & 0 & 0 & \cdots & 1
\end{array}\right)_{m \times m}
$$

So,

$$
\begin{gathered}
\int_{0}^{t} T(s) \mathrm{d} s \simeq M T(t), \\
M=\left(\begin{array}{ll}
M 1 & M 2 \\
M 1 & M 2
\end{array}\right),
\end{gathered}
$$

where $M$ is operational matrix of integration base on TFs.

Therefore, we can use the below approximation

$$
\int_{0}^{t} f(s) \mathrm{d} s \simeq \int_{0}^{t} C^{T} T(s) \mathrm{d} s \simeq C^{T} M T(t) .
$$

Now, consider the following definitions:

$$
\begin{aligned}
& \alpha(i):=(i+1)[B((i+0.5) h)-B(i h)]-\int_{i h}^{(i+0.5) h} \frac{s}{h} \mathrm{~d} B(s), \\
& \beta(i):=(i+1)[B((i+1) h)-B(i h)]-\int_{i h}^{(i+1) h} \frac{s}{h} \mathrm{~d} B(s), \\
& \gamma(i):=-i[B((i+0.5) h)-B(i h)]+\int_{i h}^{(i+0.5) h} \frac{s}{h} \mathrm{~d} B(s), \\
& \rho(i):=-i[B((i+1) h)-B(i h)]+\int_{i h}^{(i+1) h} \frac{s}{h} \mathrm{~d} B(s),
\end{aligned}
$$

let

$$
\begin{aligned}
M 1_{S} & =\left(\begin{array}{ccccc}
\alpha(0) & \beta(0) & \beta(0) & \ldots & \beta(0) \\
0 & \alpha(1) & \beta(1) & \cdots & \beta(1) \\
0 & 0 & \alpha(2) & \cdots & \beta(2) \\
\vdots & \vdots & \vdots & \ddots & \vdots \\
0 & 0 & 0 & \cdots & \beta(m-2) \\
0 & 0 & 0 & \cdots & \alpha(m-1)
\end{array}\right)_{m \times m}, \\
M 2_{S} & =\left(\begin{array}{ccccc}
\gamma(0) & \rho(0) & \rho(0) & \cdots & \rho(0) \\
0 & \gamma(1) & \rho(1) & \cdots & \rho(1) \\
0 & 0 & \gamma(2) & \cdots & \rho(2) \\
\vdots & \vdots & \vdots & \ddots & \vdots \\
0 & 0 & 0 & \cdots & \rho(m-2) \\
0 & 0 & 0 & \cdots & \gamma(m-1)
\end{array}\right)_{m \times m},
\end{aligned}
$$

and

$$
\begin{aligned}
& \int_{0}^{t} T 1(s) \mathrm{d} B(s)=M 1_{s} \cdot T 1(t)+M 1_{s} \cdot T 2(t), \\
& \int_{0}^{t} T 2(s) \mathrm{d} B(s)=M 2_{S} \cdot T 1(t)+M 2_{S} \cdot T 2(t) .
\end{aligned}
$$


Therefore,

$$
\int_{0}^{t} T(s) \mathrm{d} B(s) \simeq M_{S} \cdot T(t)
$$

where

$$
M_{S}=\left(\begin{array}{ll}
M 1_{S} & M 1_{S} \\
M 2_{S} & M 2_{S}
\end{array}\right)
$$

is stochastic operational matrix of integration in the TF domain.

So, the Itô integral of $f(t)$ is approximated with

$$
\int_{0}^{t} f(s) \mathrm{d} B(s) \simeq \int_{0}^{t} C^{T} T(s) \mathrm{d} B(s) \simeq C^{T} M_{S} T(t) .
$$

\section{Stochastic SIR model}

Most models for the transmission of infectious diseases descend from the classical SIR model of Kermack and McKendrick established in 1927, [8]. One of the most basic SIR models is

$$
\left\{\begin{array}{l}
\dot{S(t)}=\Lambda-\beta S(t) I(t)-\mu S(t) \\
I \dot{(t)}=\beta S(t) I(t)-(\mu+\epsilon+\gamma) I(t) \\
\dot{R(t)}=\gamma I(t)-\mu R(t)
\end{array}\right.
$$

where the parameters $\Lambda, \beta, \epsilon, \mu, \gamma$ are positive constants, and $S(t), I(t), R(t)$ denote the number of the individuals susceptible to the disease, number of infected members and number of members who have been removed from the possibility of infection through full immunity, respectively. Some notable features of the model: The influx of individuals into the susceptibles is given by a constant $\Lambda$, it is assumed that the natural death rates are assumed to be equal (denoted by constant $\mu$ ) and individuals in $I(t)$ suffer an additional death due to disease with rate constant $\epsilon, \beta$ and $\gamma$ represent the disease transmission coefficient and the rate of recovery from infection respectively. The basic reproduction number $R_{0}=\frac{\beta \Lambda}{\mu(\mu+\epsilon+\gamma)}$ is the threshold of the system for an epidemic to occur. If $R_{0} \leq 1$, model (38) has only the disease-free equilibrium $E_{0}=\left(\frac{\Lambda}{\mu}, 0,0\right)$ which is globally asymptotical stable. This means the disease will disappear and the entire population will become susceptible. If $R_{0}>1, E_{0}$ becomes unstable and there exists a global asymptotically stable endemic equilibrium $E^{*}=\left(\frac{\mu+\epsilon+\gamma}{\beta}, \frac{\Lambda}{\mu+\epsilon+\gamma}-\frac{\mu}{\beta}, \frac{\Lambda \gamma}{\mu(\mu+\epsilon+\gamma)}-\frac{\gamma}{\beta}\right)$ which implies the disease always remains [7].

However, in the real world, epidemic models are inevitably affected by environmental noise, which is an important component in realism. Environmental noise can provide an additional degree of realism compared to their deterministic counterparts. Stochastic models depend on the chance variations in risk of exposure, disease and other illness dynamics. In this paper, our approach to include stochastic perturbation is analogous to that of Imhof and Walcher [6]. Here we assume that stochastic perturbations are of a white noise type which are directly proportional to $S(t), I(t), R(t)$, influenced on the $\dot{S}(t), \dot{I}(t), \dot{R}(t)$ in the model (38). By this way, the model (38) will be deduced to the form:

$$
\left\{\begin{array}{l}
\mathrm{d} S(t)=(\Lambda-\beta S(t) I(t)-\mu S(t)) \mathrm{d} t+\sigma_{1} S(t) \mathrm{d} B_{1}(t), \\
\mathrm{d} I(t)=(\beta S(t) I(t)-(\mu+\epsilon+\gamma) I(t)) \mathrm{d} t+\sigma_{2} I(t) \mathrm{d} B_{2}(t), \\
\mathrm{d} R(t)=(\gamma I(t)-\mu R(t)) \mathrm{d} t+\sigma_{3} R(t) \mathrm{d} B_{3}(t),
\end{array}\right.
$$

where $B_{i}(t)$ are independent standard Brownian motions and $\sigma_{i}^{2} \geq 0$ represent the intensities of $B_{i}(t), i=$ $1,2,3$.

It is proved that the stochastic model led to extinction even though the deterministic counterpart predicts persistence. In paper [7], unique global positive solution of this model was considered. Also, the asymptotic behavior of this solution was investigated. 
Theorem 4.1 If $R_{0}=\frac{\beta \Lambda}{\mu(\mu+\epsilon+\gamma)}<1$ and the following condition is satisfied

$$
\sigma_{1}^{2}<\mu, \quad \sigma_{2}^{2}<2(\mu+\epsilon+\gamma),
$$

then for any given initial value $(S(0), I(0), R(0)) \in R_{+}^{3}$, the solution of model (39) has the property

$$
\limsup _{t \rightarrow \infty} \frac{1}{t} E \int_{0}^{t}\left[\left(s(r)-\frac{\Lambda}{\mu}\right)^{2}+I^{2}(r)+R(r)\right] d r \leq \frac{2 \sigma_{1}^{2} \Lambda^{2}}{\mu^{2} K_{1}},
$$

where

$$
K_{1}=\min \left\{2\left(\mu-\sigma_{1}^{2}\right), 2(\mu+\epsilon+\gamma)-\sigma_{2}^{2}, \frac{2(2 \mu+\epsilon+\gamma)[\mu(\mu+\epsilon+\gamma)-\beta \Lambda]}{\beta \gamma}\right\} .
$$

Proof See [7].

4.1 Application of operational matrices to solve SIR model

By integration of Eq. (39), this method is applied for the following system

$$
\left\{\begin{array}{l}
S(t)=S_{0}(t)+\int_{0}^{t} \Lambda \mathrm{d} s-\int_{0}^{t} \beta S(s) I(s) \mathrm{d} s-\int_{0}^{t} \mu S(s) \mathrm{d} s+\int_{0}^{t} \sigma_{1} S(s) \mathrm{d} B_{1}(s), \\
I(t)=I_{0}(t)+\int_{0}^{t} \beta S(s) I(s) \mathrm{d} s-\int_{0}^{t}(\mu+\epsilon+\gamma) I(s) \mathrm{d} s+\int_{0}^{t} \sigma_{2} I(s) \mathrm{d} B_{2}(s) \\
R(t)=R_{0}(t)+\int_{0}^{t} \gamma I(s) \mathrm{d} s-\int_{0}^{t} \mu R(s) \mathrm{d} s+\int_{0}^{t} \sigma_{3} R(s) \mathrm{d} B_{3}(s) .
\end{array}\right.
$$

We approximate function $S(t), S_{0}(t), I(t), I_{0}(t), R(t), R_{0}(t)$ and $\Lambda t$ by BPFs and TFs.

For convenient, we choose $\Phi(t)$, as a vector form of these functions: ( $\Psi(t)$, m-dimensional vector form of BPFs, $T(t), 2 \mathrm{~m}$-dimensional vector form of TFs and $P$ as a operational matrix of them, also, we put $P_{S}$ as a stochastic operational matrix of the discussed functions.)

$$
\begin{gathered}
S(t) \simeq \bar{S}(t)=S^{T} \Phi(t)=\Phi^{T}(t) S, \\
S_{0}(t) \simeq S_{0}^{T} \Phi(t)=\Phi^{T}(t) S_{0}, \\
I(t) \simeq \bar{I}(t)=I^{T} \Phi(t)=\Phi^{T}(t) I, \\
I_{0}(t) \simeq I_{0}^{T} \Phi(t)=\Phi^{T}(t) I_{0}, \\
R(t) \simeq \bar{R}(t)=R^{T} \Phi(t)=\Phi^{T}(t) R, \\
R_{0}(t) \simeq R_{0}^{T} \Phi(t)=\Phi^{T}(t) R_{0}, \\
\Lambda t \simeq \lambda^{T} \Phi(t)=\Phi^{T}(t) \lambda,
\end{gathered}
$$

where the vectors $S, S_{0}, I, I_{0}, R, R_{0}$ are function coefficient of $S(t), S_{0}(t), I(t), I_{0}(t), R(t), R_{0}(t)$.

Substituting $(42,43,44,45,46,47,48)$ into $(41)$, we get

$$
\left\{\begin{array}{l}
S^{T} \Phi(t)=S_{0}^{T} \Phi(t)+\lambda^{T} \Phi(t)-\beta \int_{0}^{T} S^{T} \Phi(s) \Phi^{T}(s) I \mathrm{~d} s-\mu \int_{0}^{t} S^{T} \Phi(s) \mathrm{d} s+\sigma_{1} \int_{0}^{t} S^{T} \Phi(s) \mathrm{d} B_{1}(s), \\
I^{T} \Phi(t)=I_{0}^{T} \Phi(t)+\int_{0}^{t} \beta S^{T} \Phi(s) \Phi^{T}(s) I \mathrm{~d} s-(\mu+\epsilon+\gamma) \int_{0}^{t} I^{T} \Phi(s) \mathrm{d} s+\sigma_{2} \int_{0}^{t} I^{T} \Phi(s) \mathrm{d} B_{2}(s), \\
R^{T} \Phi(t)=R_{0}^{T} \Phi(t)+\gamma \int_{0}^{t} I^{T} \Phi(t) \mathrm{d} t-\mu \int_{0}^{t} R^{T} \Phi(t) \mathrm{d} t+\sigma_{3} \int_{0}^{t} R^{T} \Phi(s) \mathrm{d} B_{3}(s) .
\end{array}\right.
$$


We replace $S^{T} \Phi(t) \Phi^{T}(t) I$ by $S^{T} \tilde{I} \Phi(t)$. So,

$$
\left\{\begin{array}{l}
S^{T} \Phi(t) \simeq S_{0}^{T} \Phi(t)+\lambda^{T} \Phi(t)-\beta S^{T} \tilde{I} P \Phi(t)-\mu S^{T} P \Phi(t)+\sigma_{1} S^{T} P_{S} \Phi(t), \\
I^{T} \Phi(t) \simeq I_{0}^{T} \Phi(t)+\beta S^{T} \tilde{I} P \Phi(t)-(\mu+\epsilon+\gamma) I^{T} P \Phi(t)+\sigma_{2} I^{T} P_{S} \Phi(t), \\
R^{T} \Phi(t) \simeq R_{0}^{T} \Phi(t)+\gamma I^{T} P \Phi(t)-\mu R^{T} P \Phi(t)+\sigma_{3} R^{T} P_{S} \Phi(t),
\end{array}\right.
$$

After replacing $\simeq$ with $=$,

$$
\left\{\begin{array}{l}
S^{T}=S_{0}^{T}+\lambda^{T}-\beta S^{T} \tilde{I} P-\mu S^{T} P+\sigma_{1} S^{T} P_{S} \\
I^{T}=I_{0}^{T}+\beta S^{T} \tilde{I} P-(\mu+\epsilon+\gamma) I^{T} P+\sigma_{2} I^{T} P_{S} \\
R^{T}=R_{0}^{T}+\gamma I^{T} P-\mu R^{T} P+\sigma_{3} R^{T} P_{S} .
\end{array}\right.
$$

By solving nonlinear system (51) we find $S, I, R$ and finally $S(t), I(t), R(t)$ of (41) are approximated.

\subsection{Numerical example}

In practice, we typically observed only a single realization of this process, a single path, out of a multitude of possible paths. The simulated sample path can then be analyzed by usual statistical method to determine how good the approximation is and in what sense it is closed to the exact solution.

In this section, we solve Eq. (41) by presented methods. Initial values $S(0)=0.7, I(0)=0.2, R(0)=0.1$ are chosen and parameters $\Lambda=0.2, \beta=0.4, \mu=0.2, \epsilon=0.1, \gamma=0.2, \sigma_{1}=0.04, \sigma_{2}=0.03, \sigma_{3}=0.02$. Then the trajectory of $S(t), I(t)$ and $R(t)$ is shown in the below Figs. $(1,2)$.
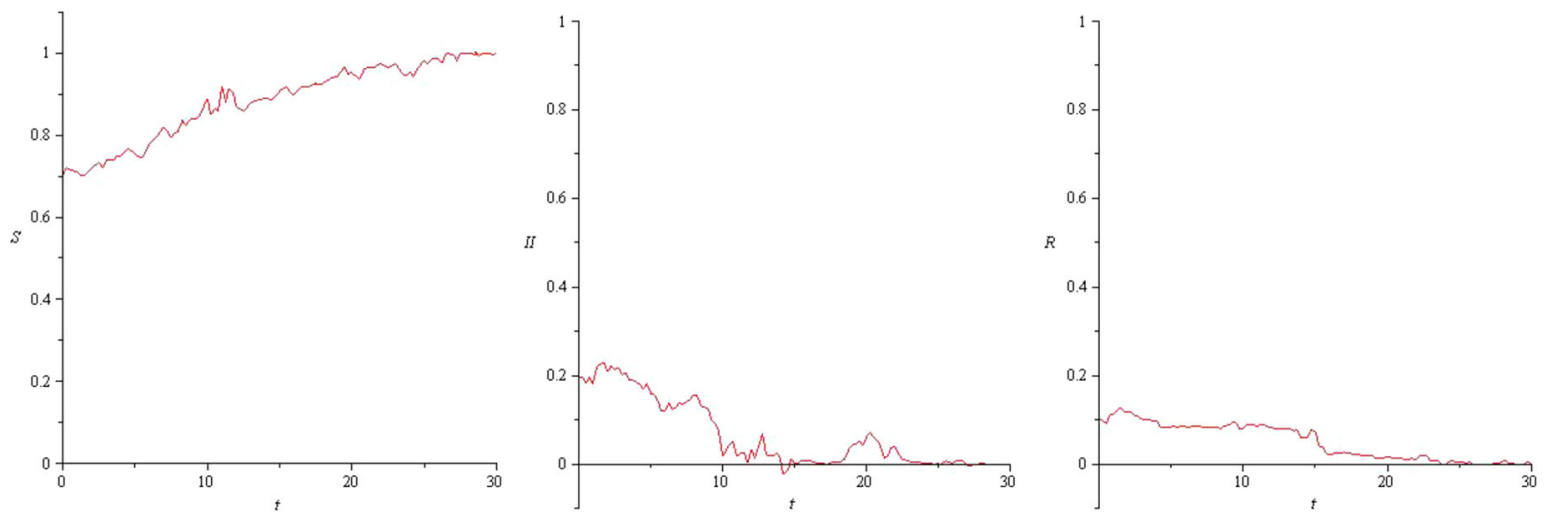

Fig. 1 Results of $S(t), I(t), R(t)$ function by BPFs, respectively
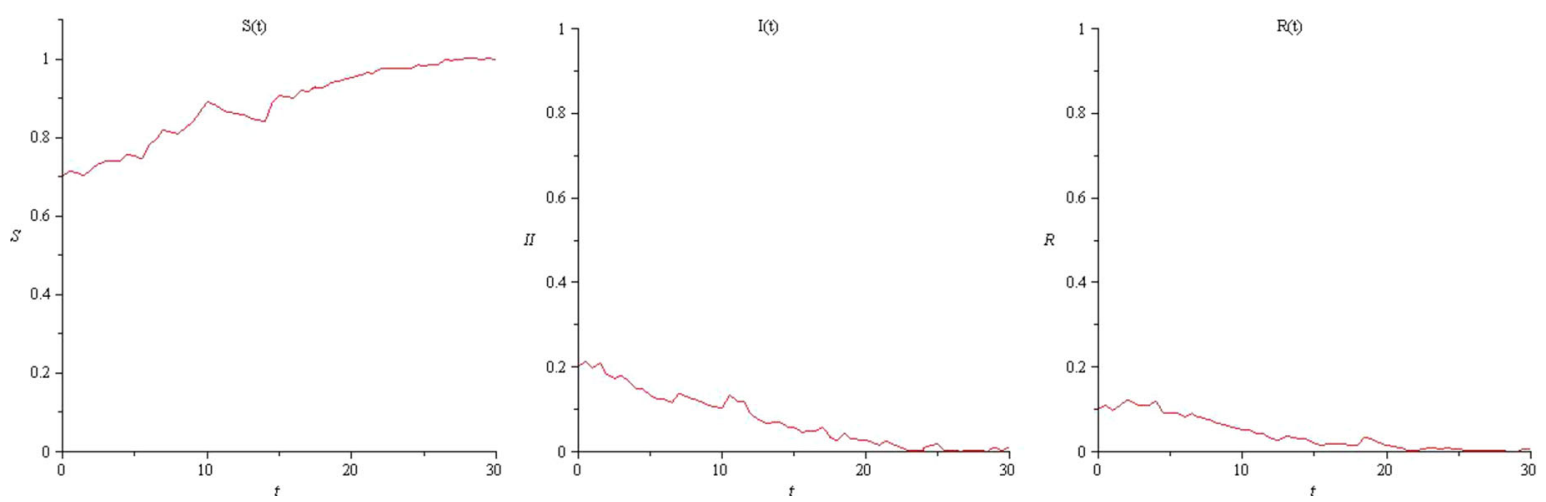

Fig. 2 Results of $S(t), I(t), R(t)$ function by TFs, respectively 
It is easy to compute that $R_{0}=0.8 \leq 1$. The computing result is in good agreement with Theorem (4.1). It is obvious that the fluctuations of the curves increase as the noise level increases. Also, we can believe the solution is stochastically asymptotically stable in the large.

Open Access This article is distributed under the terms of the Creative Commons Attribution 4.0 International License (http:// creativecommons.org/licenses/by/4.0/), which permits unrestricted use, distribution, and reproduction in any medium, provided you give appropriate credit to the original author(s) and the source, provide a link to the Creative Commons license, and indicate if changes were made.

\section{Conclusion}

Because we cannot solve some stochastic problems analytically, in this article we presented a new technique for solving systems of SVIEs numerically. We considered some operational matrices of integration. The benefits of this method are lower cost of setting up the system of equations without any integration, coupled with the low computational operation cost. Finally, convergence of TFs is faster than BPFs [9] and order of convergence is $O\left(h^{2}\right)$. These advantages make the method very simple.

\section{References}

1. Babolian, E.; Mokhtari, R.; Salmani, M.: Using direct method for solving variational problems via triangular orthogonal functions. Appl. Math. Comput. 191, 206-217 (2007)

2. Babolian, E.; Marzban, H.R.; Salmani, M.: Using triangular orthogonal functions for solving Fredholm integral equations of the second kind. Appl. Math. Comput. 201, 452-456 (2008)

3. Babolian, E.; Masouri, Z.; Hatamzadeh-Varmazyarb, S.: Numerical solution of nonlinear Volterra-Fredholm integrodifferential equations via direct method using triangular functions. Comput. Math. Appl. 58, 239-247 (2009)

4. Babolian, E.; Maleknejad, K.; Roodaki, M.; Almasieh, H.: Two-dimensional triangular functions and its applications to nonlinear 2D Volterra-Fredholm integral equations. Comput. Math. Appl. 60, 1711-1722 (2010)

5. Deb, A.; Dasgupta, A.; Sarkar, G.: A new set of orthogonal functions and its application to the analysis of dynamic systems. J. Frankl. Inst. 343, 1-26 (2006)

6. Imhof, L.; Walcher, S.: Exclusion and persistence in deterministic and stochastic chemostat models. J. Differ. Equ. 217, 26-53 (2005)

7. Jiang, D.; Yua, J.; Ji, C.; Shi, N.: Asymptotic behavior of global positive solution to a stochastic SIR model. Math. Comput. Model. 54, 221-232 (2011)

8. Kermack, W.O.; McKendrick, A.G.: Contributions to the mathematical theory of epidemics (part I). Proc. R. Soc. Lond. Ser. A. 115, 700-721 (1927)

9. Khodabin, M.; Maleknejad, K.; Hosseini Shekarabi, F.: Application of triangular functions to numerical solution of stochastic Volterra integral equations. IAENG Int. J. Appl. Math. 43(1), 1-9 (2013)

10. Klebaner, F.C.: Intoduction to Stochastic Calculus with Applications, 2nd edn. Monash University, Australia (2005)

11. Meng, X.Z.; Chen, L.S.: The dynamics of a new SIR epidemic model concerning pulse vaccination strategy. Appl. Math. Comput. 197, 528-597 (2008)

12. Maleknejad, K.; Khodabin, M.; Rostami, M.: Numerical solution of stochastic Volterra integral equations by stochastic operational matrix based on block pulse functions. Math. Comput. Model. 55, 791-800 (2012)

13. Maleknejad, K.; Rahimi, B.: Modification of block pulse functions and their application to solve numerically Volterra integral equation of the first kind. Commun. Nonlinear Sci. Numer. Simul. 16, 2469-2477 (2011)

14. Maleknejad, K.; Shahrezaee, M.; Khatami, H.: Numerical solution of integral equations system of the second kind by block pulse functions. Appl. Math. Comput. 166, 15-24 (2005)

15. Oksendal, B.: Stochastic Differential Equations. An Introduction with Applications, 5th edn. Springer-Verlag, New York (1998)

16. Roy, M.; Holt, R.D.: Effects of predation on host-pathogen dynamics in SIR models. Theor. Popul. Biol. 73, 319-331 (2008)

17. Tchuenche, J.M.; Nwagwo, A.; Levins, R.: Global behaviour of an SIR epidemic model with time delay. Math. Methods Appl. Sci. 30, 733-749 (2007)

18. Wei, H.M.; Li, X.Z.; Martcheva, M.: An epidemic model of a vector-borne disease with direct transmission and time delay. J. Math. Anal. Appl. 342, 895-908 (2008)

19. Zhang, F.P.; Li, Z.Z.; Zhang, F.: Global stability of an SIR epidemic model with constant infectious period. Appl. Math. Comput. 199, 285-291 (2008) 